\title{
Recurrence near given sets and the complexity of the Casati-Prosen map
}

\author{
Mirko Degli Esposti* Stefano Galatolo ${ }^{\dagger}$
}

May 26, 2004

\begin{abstract}
We prove a quantitative recurrence result which allow to estimate the speed of approaching of a generic orbit to the discontinuities of a map. This result is applied to the study of complexity indicators for individual orbits generated by a certain zero-entropy discontinuous maps which are related to polygonal billiards and quantum chaos.
\end{abstract}

\section{Introduction}

In this paper we prove a result that gives an estimate of the speed of approaching of a generic orbit near a given set. More precisely, we will see that the time of first entrance of a generic orbit into some neighborhood of a given set can be estimated from below by the dimension of the invariant measure near the given set. We use this result to study certain complexity indicators for individual orbits generated by the following simple (two parameter family of) discontinuous area-preserving map over the torus. The map essentially coincides with the triangle map introduced by Casati and Prosen [9] in connection with the mixing properties of flows in certain triangular billiards [8].

*Dipartimento di Matematica, Università di Bologna, Piazza di Porta S. Donato, 5, I-40127 Bologna, Italy, e-mail: desposti@dm.unibo.it

†Dipartimento di Matematica applicata, Università di Pisa, Via Bonanno 24, Pisa,Italy, e-mail: galatolo@dm.unipi.it 
More precisely, let $\theta(q)$ be the discontinuous function over the circle given by $\theta(q)=-1$ if $0 \leq q \leq 1 / 2$ and $\theta(q)=1$ otherwise.

For any $\alpha, \beta \in[0,1]$, we define the map $T_{\alpha, \beta}$ as

$$
T_{\alpha, \beta}(q, p)=(q+p+\beta+\alpha \theta(q), p+\beta+\alpha \theta(q)) \bmod 1
$$

Note that $T_{\alpha, \beta}$ can be written as the composition of three elementary maps,

$$
T_{\alpha, \beta}=B \circ R_{\beta} \circ G_{\alpha},
$$

where $B=\left(\begin{array}{ll}1 & 1 \\ 0 & 1\end{array}\right)$ is a parabolic map (a skew translation), $R_{\beta}(q, p)=$ $(q, p+\beta)$ is a translation in the $p$ direction and $G_{\alpha}$ is the discontinuous part of the dynamics $G_{\alpha}(q, p)=(q, p+\alpha \theta(q))$ this discontinuous map cuts the square along the line $x=\frac{1}{2}$ translating the two pieces in opposite directions along the line.

As it follows from the previous definition, $T_{\alpha, \beta}$ acts in a very simple way on any given set of initial conditions: there is a linear stretching, combined with a translation, due to $B \circ R_{\beta}$, and a sequence of cutting which take place on the discontinuous lines $\rho \cup \rho^{\prime}=(\{1 / 2\} \times[0,1[) \cup(\{0\} \times[0,1[)$.

The effect of the dynamics can be seen in Fig.1 and Fig.2, where the evolution of a given vertical segment is shown at different values of time and for irrational and independent values of the parameters $\alpha$ and $\beta$.
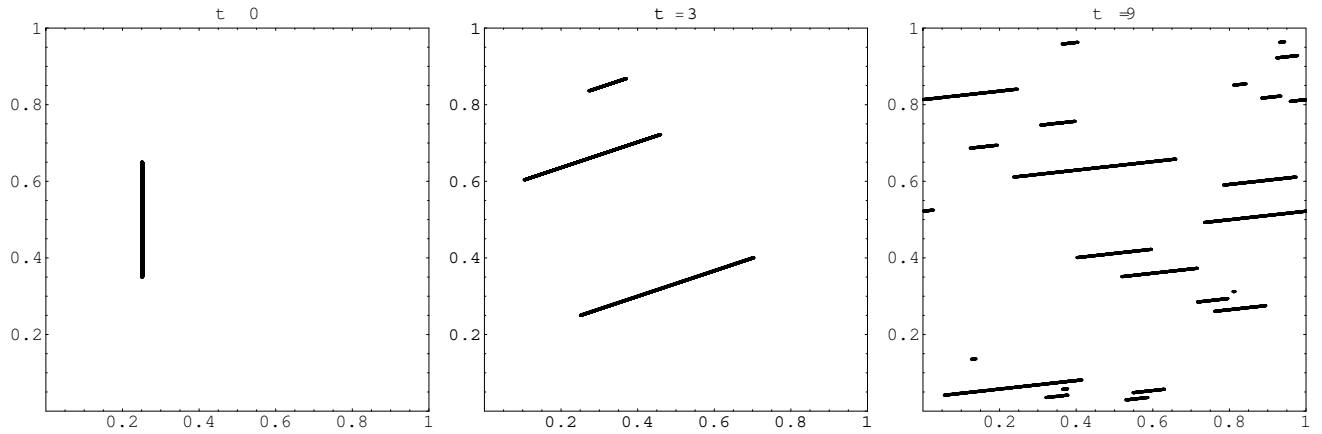

Figure 1: Evolution of a vertical segment, under the iteration of the map $T_{\alpha, \beta}$ with $\alpha=e^{-1}$ and $\beta=(1+\sqrt{5}) / 2$, at times $t=0, t=3$ and $t=9$ respectively.

The map is marginally stable i.e. initially close orbits separate linearly with time, until they are drastically separated by the discontinuity (see Sec. 4 for precise statements) moreover it has another peculiar property: absence 


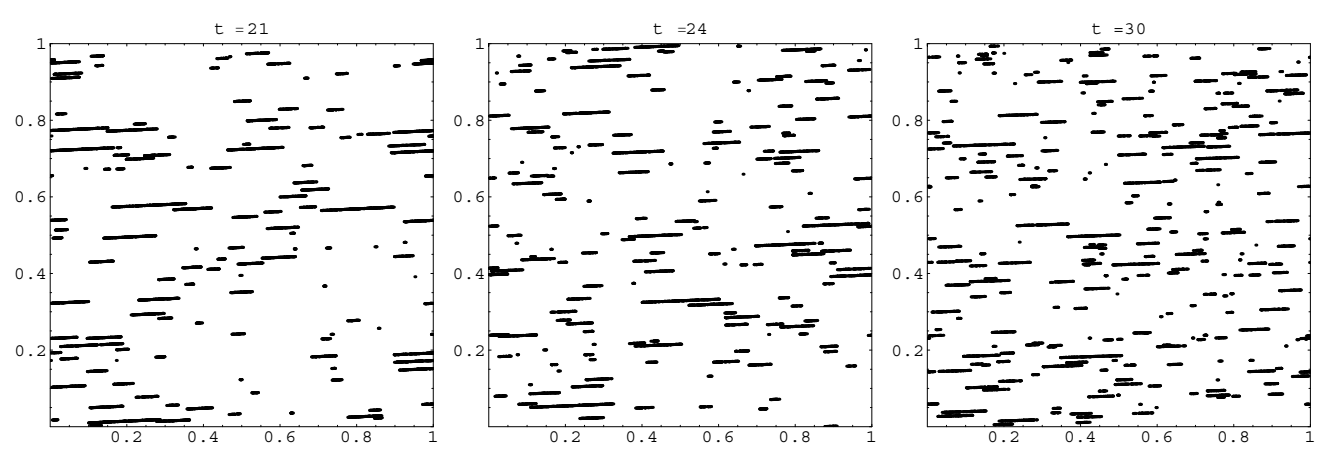

Figure 2: Evolution of a vertical segment, under the iteration of the map $T_{\alpha, \beta}$ with $\alpha=e^{-1}$ and $\beta=(1+\sqrt{5}) / 2$, at times $t=21, t=24$ and $t=30$ respectively.

of periodic orbits for irrational and incommensurable values of the parameters $\alpha$ and $\beta$, with $\beta \neq 0$. Also because of these properties, this class of maps represents a nice testing ground for some fundamental questions in the semiclassical theory of quantized discrete dynamical systems [10].

It is surprising (at least for us) that there are basically no rigorous results about ergodic or topological properties of such map. As far as we know, even ergodicity for $\alpha \neq 0$ is still not proven (even if probably true for irrational $\alpha$ 's). Here is a brief account of what it can be proven and what it can be conjectured from numerical data. First of all, if $\alpha=0, \beta \neq 0, T_{0, \beta}$ is basically a skew-translation of the torus. When $\beta$ is irrational, the dynamic is uniquely ergodic and never weak or strongly mixing $[7,11]$. In this case, the dynamics is equivalent to the one generated by interval exchange transformations [7]. However discontinuities $(\alpha \neq 0)$ seems to provide a mechanism to enforce ergodicity and establish certain mixing properties. In particular,for $\alpha \neq 0$ and $\beta \neq 0$ irrationals, the map seems ergodic and with a power law decay of correlation (with exponent close to 3/2)[9]. Finally, when $\alpha \neq 0$ is irrational and $\beta=0$, the map presents some form of ergodicity and it does not show any strong decay of correlations [9], even if a weak form of mixing is still plausible. The dynamics in the case $\beta=0$ is more regular than the generic case, and this is basically due to the very slow diffusion of the "walks" $\sum_{k=0}^{n-1} \theta\left(q_{k}\right)$ which enter in the $n$-th iterate of $T_{\alpha, 0}$. A numerical evidence of this is shown in Fig. 3, where the orbit of the same initial condition is shown at different times for $\beta=0$ and $\beta \neq 0$ respectively.

It is in any case very clear that the only process which could eventually reproduce non trivial dynamical properties (as such as the decay of correla- 

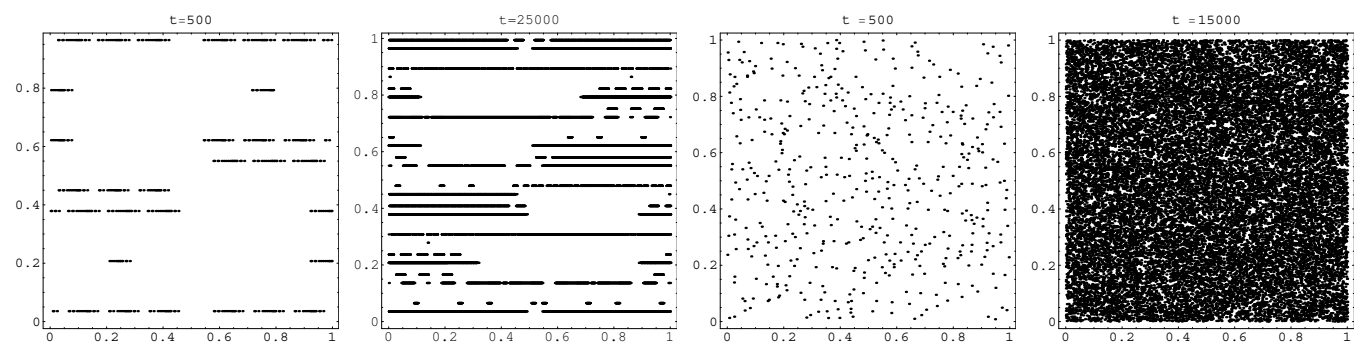

Figure 3: Orbit of a generic point under the iteration of the map $T_{\alpha, \beta}$ with $\alpha=e^{-1}$ and $\beta=0$, for $t=500$ and $t=25000$ (left two figures). Orbit of a generic point under the iteration of the map $T_{\alpha, \beta}$ with $\alpha=e^{-1}$ and $\beta=(1+\sqrt{5}) / 2$, for $t=500$ and $t=25000$ (right two figures).

tions) for these non-hyperbolic maps rely on the cutting effect produced by the discontinuities.

One of the aim of the paper is to move a first step towards the understanding of the statistics of the singularities and consequently towards the understanding of the mechanism which is responsable of the mixing behavior for such non-hyperbolic map with zero topological entropy.

In this paper we characterize the chaotic behavior of the above family of maps, giving rigorous upper bounds for the initial condition sensitivity and for the orbit complexity of the map. The results are obtained by the use of a general quantitative recurrence result (see Sec. 2, using techniques similar to the results used in [4] for the study of interval exchange transformations). Moreover we present numerical evidences that some of these upper bounds are sharp.

While the entropy of the system is a global quantity and it can be interpreted as the average information that is necessary to describe one step of the evolution of the system, the Algorithmic Information Content (AIC), due to Kolmogorov and Chaitin, is a pointwise notion: any finite string out of a finite alphabet has its own information content, independently from the context where the string appears. This is the case, for example, for strings generated by discrete dynamical systems through a given finite partition of the phase space.

In particular, the amount of information necessary to describe an orbit yield to the so called orbit complexity [5], that can be considered as a pointwise version of the entropy. In fact, this orbit complexity does in fact coincide 
almost everywhere with the entropy with respect any given invariant probability measure. More precisely, if the system has positive entropy $h>0$, the algorithmic information required to describe $n$ steps of an orbit increases as $h n+o(n)$ for almost every initial point.

Conversely, in a null entropy system, the (sublinear) asymptotic behavior of the algorithmic information describing an orbit can be considered as an indicator of weak chaos (complexity). Such an indicator can be defined in a way that it is invariant under topological conjugacy (see Sec. 3) and hence it reflect some intrinsical property of the system under consideration. Moreover (as the entropy itself does) it can be related to some dimensions and initial condition sensitivity indicators of the system [12]. Then, this complexity indicator seems to be a good candidate for an indicator replacing the entropy in the zero entropy case.

We will see that the information content of a $n$ steps orbit is low. More precisely it is lower than $C \log (n)$ (for a periodic orbit is about $\log (n)$ ). The constant $C$ depends on the coefficients $\alpha, \beta$. If we suppose that the coefficients contains a finite amount of information ( they are constructive numbers, see Definition 4) we have a sharper bound. If $\alpha, \beta$ does not contain finite information, some of this information can contribute (with the initial condition sensitivity) to the complexity of the orbit and hence $C$ could be bigger.

The paper is organized as follows. In Section 2 we prove a quantitative recurrence result that will be used to estimate the initial condition sensitivity of the Casati-Prosen maps. In Section 3, after a short introduction about the algorithmic information content we define the orbit complexity indicators. In Section 4 we use the results of Section 2 to estimate the initial condition sensitivity of the Casati-Prosen maps and in Section 5 these results are applied to estimate from above the orbit complexity of such maps.

Acknowledgment: This work has been supported by the European Commission under the Research Training Network (Mathematical Aspects of Quantum Chaos) no. HPRN-CT-2000-00103 of the IHP Programme.

\section{Quantitative recurrence near given sets}

We now define an indicator $R(x, Y)$ of recurrence near a given set $Y$. This indicator measures how faster the orbit of $x$ approaches the set $Y \subset X$. For 
our applications, $X$ is the torus, while $Y=\rho \cup \rho^{\prime}$ represents the discontinuous lines for the Casati-Prosen map. In general, given $Y \subset X$, let us define the $r$ neighborhood of $Y$ by

$$
B_{r}(Y)=\{x \in X, d(x, Y)<r\} .
$$

Let us consider the first entrance time of $x$ in $B_{r}(Y)$

$$
\tau_{r}(x, Y)=\min \left\{n, T^{n}(x) \in B_{r}(Y)\right\}
$$

By this quantity we define the following indicators measuring how fast the orbit of $x$ approaches to $Y$

$$
\underline{R}(x, Y)=\liminf _{r \rightarrow 0} \frac{\log \left(\tau_{r}(x, Y)\right)}{-\log (r)}, \bar{R}(x, Y)=\limsup _{r \rightarrow 0} \frac{\log \left(\tau_{r}(x, Y)\right)}{-\log (r)} .
$$

We remark that when $Y=x$ the above indicator is the same as the quantitative recurrence indicator $R(x)$ defined in [3].

If $\mu$ is a measure on $X, Y \subset X$, let us also consider

$$
\underline{d}_{\mu}(Y)=\limsup _{\epsilon \rightarrow 0} \frac{\log \left(\mu\left(B_{\epsilon}(Y)\right)\right)}{\log (\epsilon)} .
$$

When $Y$ is a point $\underline{d}_{\mu}(Y)$ is the lower local dimension of the measure $\mu$ with respect to the metric of $X$ at the point $Y$.

The following is a quantitative relation between our indicators of dimension and recurrence.

Theorem 1 For all $Y$ and for almost all $x, \underline{R}(x, Y) \geq \underline{d}_{\mu}(Y)$.

This theorem will be used later to calculate the initial condition sensitivity of the CP map. The proof of the following lemma is similar to Lemma 2.1 in $[4]$.

Lemma 1 Let $(X, T, \mu)$ be a measure preserving transformation, $Y \subset X$ and $\mu$ such that there is $d>0$ and $\bar{r}>0$ such that $\mu\left(B_{r}(Y)\right) \leq r^{d}$ for each $r<\bar{r}$. If $\alpha>\frac{1}{d}$ then for almost each $x \in X$ :

$$
\liminf _{n \rightarrow \infty} n^{\alpha} d\left(T^{n}(x), Y\right)=\infty
$$


Proof. Let us consider $C>0$ and the family of sets $I^{n}(Y)=T^{-n}\left(B\left(Y, C n^{-\alpha}\right)\right)$. We remark that by the assumptions stating that $\mu(B(Y, r)) \leq r^{d}$ and $\alpha>\frac{1}{d}$ then $\sum_{n} \mu\left(B\left(Y, C n^{-\alpha}\right)\right)<\infty$.

Since $T$ is measure preserving then $\sum_{n} \mu\left(I^{n}(Y)\right)<\infty$. Now each point $x$ such that $\liminf _{n \rightarrow \infty} n^{\alpha} d\left(T^{n}(x), Y\right)<C$ belongs to infinitely many sets in the family $I^{n}(Y)$. By the Borel-Cantelli lemma this implies that $x$ is contained in a zero measure set.

Proof of Theorem 1 We remark that if $(n+1)^{-\alpha} \leq r \leq n^{-\alpha}$, since $\tau_{r}(x, Y)$ is decreasing in $r$ then $\frac{\log \left(\tau_{n-\alpha}\right)}{-\log \left((n+1)^{-\alpha}\right)} \leq \frac{\log \left(\tau_{r}\right)}{-\log (r)} \leq \frac{\log \left(\tau_{(n+1)-\alpha}\right)}{-\log \left(n^{-\alpha}\right)}$, by this we can see that

$$
\liminf _{r \rightarrow 0} \frac{\log \left(\tau_{r}(x, Y)\right)}{-\log (r)}=\liminf _{n \rightarrow \infty} \frac{\log \left(\tau_{n^{-\alpha}}(x, Y)\right)}{-\log \left(n^{-\alpha}\right)} .
$$

Now Lemma 1 implies that if $n$ is big enough $\tau_{n^{-\alpha}} \geq n$ and this implies $\liminf _{n \rightarrow \infty} \frac{\log \left(\tau_{n-\alpha}(x, Y)\right)}{-\log \left(n^{-\alpha}\right)} \geq \frac{1}{\alpha}$ for each $\alpha>\frac{1}{d}$ and this implies the statement.

For the Casati-Prosen map $T_{\alpha, \beta}$, with $Y=1 / 2 \times[0,1[$ the theorem gives an upper bound that is the same both in the cases $\alpha \neq 0, \alpha=0$ (assuming $\beta \neq 0$ ). We show numerically that the bound is sharp and the speed of approaching to the discontinuity lines is the same in both cases, namely $\tau_{\epsilon}(x, Y) \sim \epsilon^{-1}$. This is shown in Fig.4 and Fig.5 for different $\epsilon$-intervals .

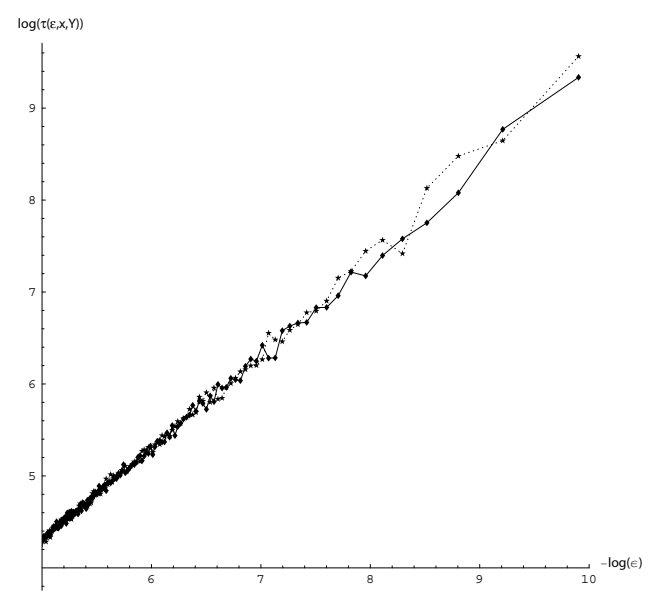

Figure 4: Plot of $-\log \epsilon,\left(\epsilon \in\left[10^{-2}, 10^{-5}\right]\right.$ versus $\log \left(\tau_{\epsilon}(x, Y)\right)$, averaged over a set of initial conditions $x \in \mathbb{T}^{2}$. Two cases are shown : $\alpha=e^{-1}, \beta=(1+\sqrt{5}) / 2$ (solid line) and $\alpha=0, \beta=(1+\sqrt{5}) / 2$ (dotted). 


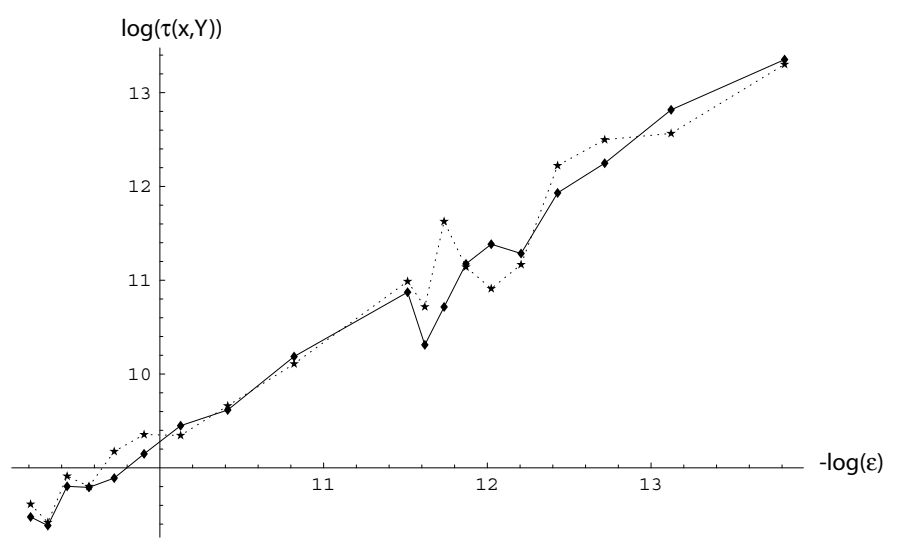

Figure 5: Plot of $-\log \epsilon, \epsilon \in\left[10^{-5}, 10^{-7}\right]$ versus $\log \left(\tau_{\epsilon}(x, Y)\right)$, averaged over a set of initial conditions $x \in \mathbb{T}^{2}$. Two cases are shown : $\alpha=e^{-1}, \beta=(1+\sqrt{5}) / 2$ (solid line) and $\alpha=0, \beta=(1+\sqrt{5}) / 2$ (dotted).

\section{$3 \quad$ Generalized orbit complexity}

We recall the definition of generalized orbit complexity that was given in [12] to provide complexity indicators for systems with 0 entropy.

The complexity of an orbit measures the quantity of information that is necessary to describe an orbit. This pointwise notion is based on a pointwise notion of information content: the Algorithmic Information Content. We now give a short recall about algorithmic information content (see [6] for more information e.g.).

Let us consider a Turing machine $A$ and a finite string $s$ written in some finite alphabet $\mathcal{A}$. Intuitively $A$ is a computer to which we can give programs to run, the programs are binary strings. Let $\Sigma$ be the set of finite binary strings. Let $p \in \Sigma$, we consider $p$ as a program to be run on the computer $A$. If we start the machine $A$ with input $p$, the computation stops and the output is $s$ we write $A(p)=s$. By this notation we emphasize the function: input $\rightarrow$ output that is naturally associated to the machine. A function is said to be recursive if its values can be computed by a Turing machine as above. Let us denote by $\ell(p)$ the length of $p$.

The Kolmogorov complexity or Algorithmic Information Content of $s$ relative to $A$ is the quantity

$$
A I C_{A}(s)=\min _{p: A(p)=s} \ell(p) .
$$


If there is no $p$ s.t. $A(p)=s$ then we put $A I C_{A}(s)=\infty$.

There are countably many Turing machines which may be computably enumerated as $A_{1}, A_{2}, \ldots$ For $n \in \mathbf{N}$ let $e_{n}$ be the $n$-th binary string in the lexicographical order $0,1,00,01,10,11,000, \ldots$ so that $\ell\left(e_{n}\right) \leq \log _{2} n$. We say that a Turing machine $U$ is universal if it can emulate any other Turing machine if appropriately programmed, more formally this can be formulate as follows: for any $n \in \mathbf{N}$ and any finite $0-1$ string $p$ we have $U\left(\hat{e}_{n} p\right)=A_{n}(p)$ where, for a given word $q$ of length $m$, we have denoted by $\hat{q}$ the word $q_{0} q_{0} q_{1} q_{1} \ldots q_{m-1} q_{m-1} 01$. This in particular means that if $A$ is any Turing machine a constant $C_{A}$ can be found so that for any finite binary string $s$ we have $A I C_{U}(s) \leq A I C_{A}(s)+C_{A}$. Then the $\mathrm{AIC}$ is independent of the choice of the universal machine $A$ up to a constant. Since we are going to consider the asymptotical behavior of the AIC for very long strings this constant is not relevant.

Let us consider a dynamical system $(X, T) . X$ is a compact metric space and $T$ is a Borel map $X \rightarrow X$. Many nice results hold when $T$ is continuous, the next definition does not need continuity and is then suited also for the CP map.

Let us consider a finite open cover $\beta=\left\{B_{0}, B_{1}, \ldots, B_{N-1}\right\}$ of $X$. We use $\beta$ to code the orbits of $(X, T)$ into a set of infinite strings. A symbolic coding of the orbits of $X$ with respect to the cover $\beta$ is a string listing the sets $B_{i_{1}}, \ldots, B_{i_{n}}$ visited by the orbit of $x$ during the iterations of $T$. Since the sets $B_{i}$ may have non empty intersection then an orbit can have more than one possible coding. More precisely, if $x \in X$ let us define the set of symbolic orbits of $x$ with respect to $\beta$ as:

$$
\varphi_{\beta}(x)=\left\{\omega \in\{0,1, \ldots, N-1\}^{\mathbf{N}}: \forall n \in \mathbf{N}, T^{n}(x) \in B_{\omega(n)}\right\} .
$$

The set $\varphi_{\beta}(x)$ is the set of all the possible codings of the orbit of $x$ relative to the cover $\beta$.

Definition 1 The information content of $n$ steps of the orbit of $x$ with respect to $\beta$ is defined as

$$
K(x, T, \beta, n)=\min _{\omega \in \varphi_{\beta}(x)} A I C_{U}\left(\omega^{n}\right)
$$

where $\omega^{n}$ is the string containing the first $n$ digits of $\omega$. 
By [5], when a system has an invariant measure $\mu$ with positive entropy we have $K(x, T, \beta, n)=h(\beta) n+o(n)$, where $h(\beta)$ tends to $h_{\mu}$ as the diameter of $\beta$ tends to 0 . Hence in positive entropy systems the information increases linearly. In a zero entropy system the information increases with a sublinear asymptotic behavior. To be able to distinguish between all the possible sublinear asymptotic behaviors of the $K(x, T, \beta, n)$ as $n$ increases we compare it with a function $f$ whose asymptotic behavior is known. For each monotonic function $f(n)$ with $\lim _{n \rightarrow \infty} f(n)=\infty$ we consider

Definition 2 The complexity of the orbit of $x \in X$ relative to $f$ and $\beta$ is defined as:

$$
K^{f}(x, T, \beta)=\limsup _{n \rightarrow \infty} \frac{K(x, T, \beta, n)}{f(n)} .
$$

For the Casati-Prosen map we are interested mainly to the case where $f(n)=\log (n)$.

Taking the supremum over the set of all finite open covers $\beta$ of the metric space $X$ it is possible to get rid of the dependence of our definition on the choice of the cover $\beta$ and define the complexity of the orbit of $x$ :

Definition 3 The complexity of $x$ with respect to $f$ is defined as

$$
K^{f}(x, T)=\underset{\beta \in\{\text { Finite open covers }\}}{\text { sup }} K^{f}(x, T, \beta) .
$$

This definition associates to a point belonging to $X$ and a function $f$ a real number which is a measure of the complexity of the orbit of $x$ with respect to the asymptotic behavior of $f$.

An interesting feature of this definition is that it is invariant by topological conjugation: if the dynamical systems $(X, T)$ and $(Y, S)$ are topologically conjugate, $\pi: X \rightarrow Y$ is the conjugating homeomorphism, and $\pi(x)=y$ then $\forall f, K^{f}(x, T)=K^{f}(y, S)$ (see [12]).

Another interesting feature of orbit complexity is that it can be numerically estimated by the use of suitable data compression algorithms (see e.g. [1]). This gives a tool for the analisys of dynamical time series.

In the following we will give a rigorous estimation of $K^{\log }(x)$ for the orbits generated by the Casati-Prosen map. 


\section{Initial condition sensitivity of the Casati- Prosen map}

The Casati-Prosen map is sensitive to initial conditions, for example in the formal sense of Devaney (see e.g. [2]). At an intuitive level the reason for this is that nearby starting orbits are slowly separated by the effect of the skew translation until they are drastically separated by the discontinuity. We now consider a quantitative indicator of initial condition sensitivity.

The indicator is constructed in a way that can detect this weak form of initial condition sensitivity. Its value will be estimated by the use of Theorem 1. Let us consider the following set:

$$
B(n, x, \epsilon)=\left\{y \in X: d\left(T^{i}(y), T^{i}(x)\right) \leq \epsilon \forall i \text { s.t. } 0 \leq i \leq n\right\}
$$

$B(n, x, \epsilon)$ is the set of points "following" the orbit of $x$ for $n$ steps at a distance less than $\epsilon$. As the nearby starting orbits of $(X, T)$ diverges the set $B(n, x, \epsilon)$ will be smaller and smaller as $n$ increases. The speed of decreasing of the size of this set considered as a function of $n$ will be a measure of the sensitivity of the system to changes on initial conditions on a neighborhood of $x$. As a measure for the size of $B(n, x, \epsilon)$ we consider the radius of the biggest ball with center in $x$ contained in $B(n, x, \epsilon)$, i.e.

$$
r(x, n, \epsilon)=\sup _{B_{r}(x) \subset B(n, x, \epsilon)} .
$$

To measure the speed of decreasing of this size we measure how faster $-\log (r(x, n, \epsilon))$ increases as $n$ increases. This is, in a certain sense an estimation from above of the initial condition sensitivity (see [12]). To have consistent notations with the definition of $K^{f}$ let us consider an $f$ as above and let us define $r_{\epsilon}^{f}: X \rightarrow \mathbf{R}$

$$
r_{\epsilon}^{f}(x)=\limsup _{n \rightarrow \infty} \frac{-\log (r(x, n, \epsilon))}{f(n)} .
$$

Since $r_{\epsilon}^{f}(x)$ is a monotone function with respect to $\epsilon$ we can define

$$
r^{f}(x)=\sup _{\epsilon \in \mathbf{R}^{+}} r_{\epsilon}^{f}(x) .
$$




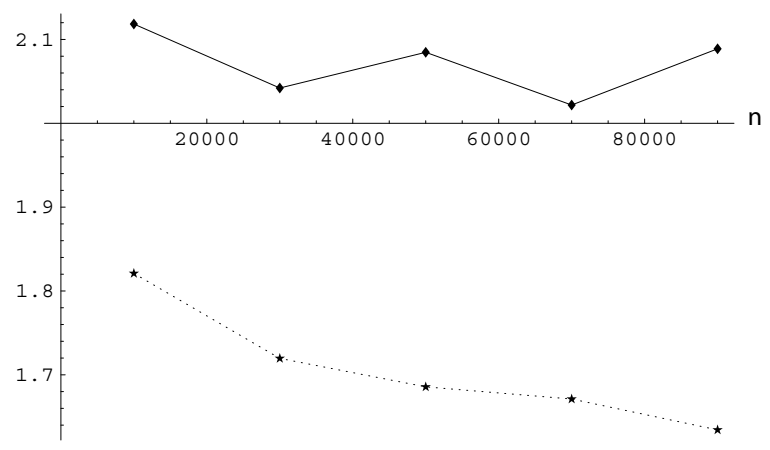

Figure 6: plot of $-\frac{\log r(x, n, \epsilon)}{\log n}$ versus $n$, for two given initial conditions $x \in \mathbb{T}^{2}$, $\epsilon=0.0005$, where $\alpha=1 / e, \beta=(\sqrt{5}+1) / 2($ solid $)$ and $\alpha=0, \beta=(\sqrt{5}+1) / 2$ (dashed).

Now let us estimate $r^{\log }(x)$ for the CP map. Let us consider two nearby starting initial conditions $x$ and $y$. As said before the orbits of $x$ and $y$ will separate by the effect of the skew translation and by the effect of the discontinuity. In particular, the combination of the linear separation induced by the regular part of the dynamics, together with the estimate for the recurrence close to the discontinuous lines, $\tau_{\epsilon}(x, Y) \sim \epsilon^{-1}$, leads to the estimate $r_{\epsilon}^{\log }(x) \sim 2$, for $T_{\alpha, \beta}$ with $\alpha, \beta \notin \mathbb{Q} \cup(0)$. On the other side, this behavior must be compared with the asymptotic $r_{\epsilon}^{\log }(x) \sim 1$ that we have for the continuous skew translation $F_{0, \beta}$. This is shown numerically in Fig. 6, whereas the effect of the discontinuity can be rigorously estimated by the use of Theorem 1 , which leads to:

Theorem 2 For Lebesgue almost each $x \in X r^{\log }(x) \leq 2$.

Proof. First it is easy to remark that if $Y=\rho \cup \rho^{\prime}$ is the discontinuity set of the map $\underline{d}_{\mu}(Y)=1$. Let $\alpha<1$, let $x \in X$ be such that (by theorem 1 ) $\liminf \operatorname{in}_{n \rightarrow \infty} n^{\alpha} d\left(T^{n}(x), Y\right)=\infty$. Let also suppose that the orbit of $x$ never meet $Y$. We can suppose that for all $n n^{\alpha} d\left(T^{n}(x), Y\right) \geq c>0$

Let us consider an $y$ such that $\forall i \leq n$

$$
d\left(T_{\alpha, \beta}^{i}(x), T_{\alpha, \beta}^{i}(y)\right) \leq \frac{c}{2} i^{-\alpha} .
$$

Then the orbits of $x$ and $y$ are not separated by the discontinuity at the $n+1$ step. This is true because the orbit of $x$ will stay far away (more than $\frac{c}{2} n^{-\alpha}$ ) enough from $Y$. 
Now let us consider the effect of the skew translation and estimate how near we need to start from $x$ to assure that Eq. 2 hold.

If $y \in B\left(x, \frac{c}{2} n^{-\alpha-1}\right)$ and the orbit of $x, y$ are not separated by the discontinuity then Eq. 2 holds. Indeed after $m$ steps, if the orbit of $x$ and $y$ are not separated by the discontinuity but only by the effect of the skew translation we have that $d\left(T^{m}(x), T^{m}(y)\right) \leq \frac{c}{2} m n^{-\alpha-1}$ When $m=n$ we have the desired inequality.

Then for almost each $x$, if $n$ is big enough $B(x, n, \epsilon)$ contains $B\left(x, \frac{c}{2} n^{-\alpha-1}\right)$. This holds for each $\alpha>1$ and then easily implies the statement.

\section{Complexity of the orbits of the Casati-Prosen map}

Now we will deduce an estimation about orbit complexity by the estimation on initial condition sensitivity given before, for this we need some technical tools. A real number is constructive if it can be approximated at any accuracy by an algorithm.

Definition 4 (Constructive numbers) A number $z \in \mathbf{R}$ is said to be constructive if there is an algorithm $P_{z}(n): \mathbf{N} \rightarrow \mathbf{Q}$ such that $P_{z}(n)=q$ implies $|q-z|<2^{-n}$.

Now let us consider the map $T_{\alpha, \beta}$ with $\alpha$ and $\beta$ constructive numbers. In [12] general relations are proved between orbit complexity and initial condition sensitivity. These relations are proved assuming that the dynamics is given by a continuous constructive map. However the above cited results can be extended to the CP map, as follows

Theorem 3 In the Casati-Prosen map with constructive coefficients the following relation holds for Lebesgue almost each point $x$ of the torus

$$
K^{\log }(x) \leq 2 r^{\log }(x)+1
$$

Proof. In this proof we use some results and techniques from [12] related to computable structures and the relation between sensitivity and complexity. We describe a construction similar to what is done in [12] section 8.1, in order

to apply theorem 31 of the cited paper to a discontinuous case. Due to lack of 
space we will not define here all the technical instruments about computable structures that are needed for the proof. We refer the reader to [12].

Let us consider $Z=\cup_{i \in \mathbf{Z}} T^{i}(Y)$, where $Y$ is the discontinuity set of $T_{\alpha, \beta}$. Let $X^{\prime}=[0,1] \times[0,1] \backslash Z$. $X^{\prime}$ is then a full measure set in $[0,1] \times[0,1]$.

On the space $X^{\prime}$ it can be considered the computable structure induced by the standard computable structure of $X$. Indeed, let us consider an interpretation ([12] Definition 3) $I: \Sigma \rightarrow X$ constructed in the following way: first let us consider a recursive encoding of $\mathbf{Q} \times \mathbf{Q}$ with strings, i.e. an $\left(\begin{array}{l}l_{1} \\ l_{2}\end{array}\right): \Sigma \rightarrow \mathbf{Q} \times \mathbf{Q}$, then consider $I(s)=\left(l_{1}(s)+z(\bmod 1), l_{2}(s)+z(\bmod 1)\right)$ where $z$ is a constructive irrational that is incommensurable with $\alpha$ and $\beta$.

On this (non compact) space $X^{\prime}$ the map $T_{\alpha, \beta}$ is constructive (in the sense of [12] definition 9). Theorem 31 of [12] and the fact (Theorem 19 of [12]) that in a compact space $([0,1] \times[0,1])$ the orbit complexity with respect to a computable structure is equivalent to the orbit complexity with respect to open covers (as it is defined in this paper) implies that for each $x \in X^{\prime}$ and hence for almost each $x \in[0,1] \times[0,1]$

$$
K^{\log }(x) \leq \bar{d}_{B}\left(X^{\prime}\right) r^{\log }(x)+1
$$

were $\bar{d}_{B}$ is the upper box counting dimension of $X^{\prime}$, which is 2 and by this we have the statement

From the previous statement and Theorem 2 we finally have an estimation about the generalized orbit complexity of the CP map when the coefficients are constructive numbers

Corollary 1 In the Casati-Prosen map, with constructive coefficients, for Lebesgue almost each $x, K^{\log }(x) \leq 3$.

This implies that if the map has constructive coefficients for almost all points the information that is necessary to describe $n$ steps of an orbit increases at most as $3 \log (n)$ modulo lower order terms.

If the map has no constructive coefficients an estimation of the information content of the orbits is still possible.

Theorem 4 In the Casati-Prosen map, for Lebesgue almost each $x$,

$$
K^{\log }(x) \leq 11
$$


Lemma 2 Let us consider the CP map $T_{\alpha, \beta}$, with $\alpha, \beta$ constructive numbers, let $z$ be a constructive number that is not commensurable with $1, \alpha$ and $\beta$, let $x=\left(\begin{array}{l}q_{1}+z \\ q_{2}+z\end{array}\right)$ with $q_{1}, q_{2} \in \mathbf{Q}$. Then, there is a program $P$ that calculates a recursive function $A: \mathbf{N} \times \mathbf{Q} \times \mathbf{Q} \times \mathbf{Q} \rightarrow \mathbf{Q} \times \mathbf{Q}$ such that $d\left(A\left(n, \epsilon, q_{1}, q_{2}\right), T_{\alpha, \beta}^{n}(x)\right)<\epsilon$ and the length of $P$ satisfies $|P| \leq C+\left|P_{\alpha}\right|+$ $\left|P_{\beta}\right|$ (see Def. 4).

Proof. If $x$ is as above, since $z$ is irrational $x$ is not on the discontinuity set. It is easy to see that with the knowledge of $\alpha$ and $\beta$ it is possible to calculate with an algorithm $T_{\alpha, \beta}(x)$ with an arbitrary precision. Since $z$ is not commensurable with $1, \alpha$ and $\beta$, then $T_{\alpha, \beta}(x)$ is not on the discontinuity line (and the whole orbit of $x$ never meet the line). Since $T_{\alpha, \beta}$ is continuous in a neighborhood of $x$ by approximating $T_{\alpha, \beta}(x)$ with a sequence of points $x_{1}=$ $\left(\begin{array}{c}q^{1}{ }_{1}+z \\ q^{1}{ }_{2}+z\end{array}\right), x_{2}=\left(\begin{array}{c}q^{2}{ }_{1}+z \\ q^{2}{ }_{2}+z\end{array}\right), \ldots$ converging to $T_{\alpha, \beta}(x)$ and calculating $T_{\alpha, \beta}\left(x_{1}\right), T_{\alpha, \beta}\left(x_{2}\right) \ldots$ with a greater and greater precision we can calculate a sequence of points that converges to $T_{\alpha, \beta}^{2}(x)$, hence we can calculate $T_{\alpha, \beta}^{2}(x)$ with arbitrary precision. By iterating this step it is easy to see that $T_{\alpha, \beta}^{m}(x)$ can be calculated with arbitrary precision by an algorithm (the algorithm $A$ ). We remark that varying $\alpha$ and $\beta$ the main part of the procedure remain the same, we only need the information that is necessary to construct $\alpha$ and $\beta$ with arbitrary precision, that is $\left|P_{\alpha}\right|+\left|P_{\beta}\right|$.

Lemma 3 Let us consider $T_{\alpha_{1}, \beta_{1}}, \epsilon<2 \alpha_{1}$ and let $d>1$. For almost each $x$ there is a $c$ such that if $\alpha_{2}$ and $\beta_{2}$ are such that $\left|\alpha_{1}-\alpha_{2}\right|<\frac{c}{4 n^{d+2}}$ and $\left|\beta_{1}-\beta_{2}\right|<\frac{c}{24 n^{d+2}}$ then it holds $\forall i \leq n+1 d\left(T_{\alpha_{1}, \beta_{1}}^{i}(x), T_{\alpha_{2}, \beta_{2}}^{i}(x)\right) \leq \frac{\epsilon}{4}$.

Proof. Let us consider some $d>1$, like in the proof of Theorem 2. Let us consider an $x$ such that there is a $c>0$ s.t. for each $n, n^{d} d\left(T_{\alpha_{1}, \beta_{1}}^{n}(x), Y\right) \geq$ $c>0$ and $n^{d} d\left(T_{\alpha_{2}, \beta_{2}}^{n}(x), Y\right) \geq c>0$. By theorem 1 this assumption can be made for almost each $x$.

Let $n$ be such that $c n^{-d}<\epsilon$. If $d\left(T_{\alpha_{2}, \beta_{2}}^{n}(x), T_{\alpha_{1}, \beta_{1}}^{n}(x)\right)<\frac{c}{4} n^{-d}<\frac{\epsilon}{4}$ then at the next step the two orbits cannot be separated by the discontinuity and they will keep to stay at a distance less than $\epsilon$.

Let us hence suppose that the two orbits are not separated by the discontinuity for $n$ steps. Let us use the following notations: $x=\left(\begin{array}{c}x_{0} \\ y_{0}\end{array}\right)$, 
$T_{\alpha_{1}, \beta_{1}}(x)=\left(\begin{array}{c}x_{1} \\ y_{1}\end{array}\right), T_{\alpha_{2}, \beta_{2}}(x)=\left(\begin{array}{c}x_{1}^{\prime} \\ y_{1}^{\prime}\end{array}\right), T_{\alpha_{1}, \beta_{1}}{ }^{i}(x)=\left(\begin{array}{c}x_{i} \\ y_{i}\end{array}\right), T_{\alpha_{2}, \beta_{2}}{ }^{i}(x)=$ $\left(\begin{array}{c}x_{i}^{\prime} \\ y_{i}^{\prime}\end{array}\right)$, and finally $\Delta x_{i}=\left|x_{i}-x_{i}^{\prime}\right|$ and $\Delta y_{i}=\left|y_{i}-y_{i}^{\prime}\right|$. With these notations we have for each $n \geq 1$ the following $\left\{\begin{array}{c}\Delta y_{n}=n \Delta y_{0} \\ \Delta x_{n}=\Delta x_{n-1}+\Delta y_{n-1}\end{array}\right.$.

By this we can estimate $\Delta y_{1} \leq\left|\alpha_{1}-\alpha_{2}\right|+\left|\beta_{1}+\beta_{2}\right|$ and $d\left(T_{\alpha_{1}, \beta_{1}}^{i}, T_{\alpha_{2}, \beta_{2}}^{i}\right) \leq$ $\Delta y_{1}\left(i+\frac{i(i+1)}{2}\right) \leq 3 \Delta y_{1} i^{2}$ for each $i \leq n$.

Hence if $\Delta y_{1} \leq\left|\beta_{1}-\beta_{2}\right|+\left|\alpha_{1}-\alpha_{2}\right| \leq \frac{c}{12 n^{d+2}}$ then for each $i \leq n$ $d\left(T_{\alpha_{2}, \beta_{2}}^{n}(x), T_{\alpha_{1}, \beta_{1}}^{n}(x)\right)<\frac{\epsilon}{4}$. Recalling that this holds for almost each $x$ we have the statement

Proof of theorem 4. After the estimation (Theorem 2 ) on $r$ we have that for each $d>1$ there is a $C$ such that $B(x, n, \epsilon)$ contains a ball with radius $\frac{C}{n^{d+1}}$. In such a ball there is a point of the type $y=\left(\begin{array}{c}q_{1}+z \\ q_{2}+z\end{array}\right)$ (as in Lemma 2 ) and the binary expansion of $q_{1}$ and $q_{2}$ are $q_{1}=0 . v_{1} v_{2} \ldots v_{l}, q_{1}=0 . w_{1} w_{2} \ldots w_{l^{\prime}}$ with $\max \left(l, l^{\prime}\right)<-\log \left(\frac{C}{n^{d+2}}\right)$ (that is about $4 \log (n)$ bits for both strings).

Let us consider a cover $U$ (see definition of orbit complexity) of $X$ made of balls with rational radius and rational center. Suppose that the Lebesgue constant of the cover is $\epsilon$. A program to give a symbolic orbit for the point $x$ for $n$ steps with respect to $U$ is the following.

0 ) the program contains strings to codify: i) the point $y$ as above, ii) a couple of rationals $\alpha_{2}, \beta_{2}$ such that $\left|\alpha-\alpha_{2}\right|+\left|\beta-\beta_{2}\right|<\frac{c}{12 n^{d+2}}$ (that is about $8 \log (n)$ bits, we remark that here $c$ is constant when $y$ is fixed), a string codifying $n$.

1) Applying lemma 2 to the approximate map $T_{\alpha_{2}, \beta_{2}}$ the program calculates a sequence of points $p_{1} \ldots p_{n}$ following the orbit of $y$ at a distance less than $\frac{\epsilon}{4}$ for $n$ steps. This sequence of points will follow the real orbit of $x$ at a distance less than $\frac{\epsilon}{2}$.

2) by calculating in which ball of the cover $U$ is the point $p_{i}$ for $1 \leq i \leq n$ the program outputs a symbolic orbit of $x$ with respect to $U$.

The program calculates the symbolic orbit of the point $x$ w.r.t $U$ for the map $T_{\alpha_{1}, \beta_{1}}$. But since the orbit of $x$ w.r.t $T_{\alpha, \beta}$ is at a distance less than $\frac{\epsilon}{2}$ then the symbolic orbit is the same.

It is clear that in the above program (asymptotically, as $n$ increases), the greater part of the information is contained in the four initial strings. They indeed needs less than $11 \log (n)+C$ bits to be given while the remaining 
part of the procedure has constant length with respect to $n$.

\section{References}

[1] Argenti, F; Benci, V; Cerrai, P; Cordelli, A; Galatolo, S; Menconi, G. Information and dynamical systems: a concrete measurement on sporadic dynamics. Classical and quantum complexity and nonextensive thermodynamics (Denton, TX, 2000). Chaos Solitons Fractals 13 (2002), no. 3, 461-469.

[2] Banks, J.; Brooks, J.; Cairns, G.; Davis, G.; Stacey, P. On Devaney's definition of chaos. Amer. Math. Monthly 99 (1992), no. $4,332-334$.

[3] L Barreira, B SAussol, Hausdorff dimension of measures via Poincaré recurrence, Commun. Math. Phys., 219 (2001), 443-463.

[4] C. Bonanno, S. Galatolo and S. Isola, Recurrence and algorithmic information Nonlinearity 17 (2004) 1057-1074.

[5] A Brudno, Entropy and the complexity of trajectories of dynamical systems, Russ. Math. Surv. 2 (1983), 127-151 (English transl.).

[6] Chaitin G.J. Information, randomness and incompleteness. Papers on algorithmic information theory. World Scientific, Singapore 1987.

[7] I.P. Cornfeld, S.V. Fomin and Ya. G. Sinai, Ergodic Theory, Springer-Verlag, New York (1982).

[8] G. Casati and T. Prosen, Mixing property of triangular billiards, Physical Review Letters, 83, n.23 (1999), 4729-4732.

[9] G. Casati and T. Prosen, The triangle map: a model of quantum chaos, , Physical Review Letters, 85, (2000), 4261.

[10] M. Degli Esposti and S. Graffi (Editors), The Mathematical Aspects of Quantum Maps, Lecture Notes in Physics 618, (2003).

[11] H. Furstenberg, Strict Ergodicity and Trasformation of the Torus,Amer. J. Math. 83, (1961), 573. 
[12] S Galatolo, Complexity, initial condition sensitivity, dimension and weak chaos in dynamical systems Nonlinearity 16, Number 4, July 2003, 1219-1239. 